\title{
Influence of Cholesterol on Catecholamine Release from the Fusion Pore of Large Dense Core Chromaffin Granules
}

\author{
Nan Wang, Christina Kwan, Xiandi Gong, Elena Posse de Chaves, Amy Tse, and Frederick W. Tse \\ Department of Pharmacology and Center for Neuroscience, University of Alberta, Edmonton, Alberta T6G 2H7, Canada
}

Changes in cellular cholesterol can affect exocytosis, but the influence of cholesterol in fusion pore kinetics is unclear. Using carbon fiber amperometry, we monitored quantal catecholamine release from rat chromaffin cells. To bypass any possible effect of cholesterol perturbation on ion channels or the colocalization of voltage-gated $\mathrm{Ca}^{2+}$ channels with sites of exocytosis, exocytosis was stimulated via uniform elevation of cytosolic $\left[\mathrm{Ca}^{2+}\right]$ (with whole-cell dialysis of a $\mathrm{Ca}^{2+}$-buffered solution). Under this condition, alterations of cellular cholesterol affected neither the mean number of amperometric events triggered per cell nor their quantal size and the kinetics of their main spike (which reflects the rapid release during and after rapid fusion pore dilation). In contrast, the reduction of cellular cholesterol shortened the "prespike foot" signals (which reflect the leakage of catecholamine via a semi-stable fusion pore) and reduced the proportion of "stand-alone foot" signals (which reflect the release via a flickering fusion pore that may close before it dilates significantly), whereas an oversupply of cholesterol had opposite effects. Acute extraction of cholesterol from the cytosol (via whole-cell dialysis of a cholesterol extractor) also shortened the prespike foot signals and reduced the proportion of stand-alone foot signals, but acute extracellular application of cholesterol extractor or "soluble" cholesterol had no effect. Our data raise the possibility that cholesterol molecules, particularly those in the cytoplasmic leaflet, helps to constrain the narrow waistline of a semi-stable fusion pore while it is flickering or before it starts to dilate rapidly.

\section{Introduction}

The cholesterol-rich "lipid rafts" of secretory cells and synapses have been suggested to play important roles in transmitter and hormone release because such microdomains of the plasma membrane can concentrate proteins important for regulating exocytosis (Salaun et al., 2004) and may influence their colocalization with voltage-gated $\mathrm{Ca}^{2+}$ channels (VGCCs) and G-proteins (Taverna et al., 2004, 2007). Lowering of cellular cholesterol level has been reported to increase (Xia et al., 2004, 2007) or decrease (Lang et al., 2001; Ohara-Imaizumi et al., 2004; Zamir and Charlton, 2006) hormone or neurotransmitter release from different cell types by affecting multiple sites in the excitationsecretion pathways. For example, cholesterol extraction has been shown to alter the function of ion channels (Xia et al., 2004, 2007; Zamir and Charlton, 2006), the balance of spontaneous and evoked release of synaptic vesicles (Zamir and Charlton, 2006; Wasser et al., 2007), as well as the proper clustering of SNARE (soluble $\mathrm{N}$-ethylmaleimide-sensitive factor attachment protein receptor) proteins (Lang et al., 2001). Moreover, studies on the membrane fusion of sea urchin cortical granules (Churchward et al., 2005; Churchward et al., 2008) and the fusion mediated by the viral protein hemagglutinin (HA) (from the influenza) (Razinkov et al., 1998; Razinkov and Cohen, 2000; Biswas et al.,

Received Aug. 14, 2009; revised Jan. 15, 2010; accepted Feb. 3, 2010.

This work was supported by grants from the Canadian Institute of Health Research. We thank Jihuan Yin for assistance in statistics analysis and Profs. Ronald McElhaney and Jean Vance for discussions.

Correspondence should be addressed to Frederick W. Tse, 9-70 Medical Sciences Building, Department of Pharmacology, University of Alberta, Edmonton, Alberta T6G 2H7, Canada. E-mail: fred.tse@ualberta.ca.

DOI:10.1523/JNEUROSCI.4000-09.2010

Copyright $\odot 2010$ the authors $\quad 0270-6474 / 10 / 303904-08 \$ 15.00 / 0$
2008) suggested that cholesterol is a key structural component of the fusion pore. A recent study on PC12 cells has also shown that cholesterol extraction reduced the number of amperometric signals triggered by the elevation of extracellular $\left[\mathrm{K}^{+}\right]$and retarded fusion pore kinetics and vesicular movement (Zhang et al., 2009). However, cholesterol reduction has been reported to affect VGCCs (Xia et al., 2004, 2008; Vikman et al., 2009), and the magnitude of cytosolic $\left[\mathrm{Ca}^{2+}\right]\left(\left[\mathrm{Ca}^{2+}\right]_{\mathrm{i}}\right)$ elevation has been shown to influence the quantal size $(\mathrm{Q})$ (the amount of catecholamines released per vesicle), as well as the mode of exocytosis (Elhamdani et al., 2001, 2006). Most importantly, the kinetics of amperometric signal is strongly influenced by $\mathrm{Q}$ (Sombers et al., 2004; Tang et al., 2007). Thus, it is not clear whether some of the effects observed in the study on PC12 cells (Zhang et al., 2009) are secondary to changes in the depolarization-triggered $\mathrm{Ca}^{2+}$ signal.

Here, we examined whether changes in cellular cholesterol level affect catecholamine release from large dense core granules (LDCGs) of rat chromaffin cells at sites distal to elevation in $\left[\mathrm{Ca}^{2+}\right]_{\mathrm{i}}$. Exocytosis was stimulated directly via whole-cell dialysis of a $\mathrm{Ca}^{2+}$-buffered solution $\left(\left[\mathrm{Ca}^{2+}\right]\right.$ of $\left.\sim 0.5 \mu \mathrm{M}\right)$, and carbon fiber amperometry was used to examine the detailed kinetics of catecholamine release from individual LDCGs. Overall, our results suggest that cholesterol molecules, particularly those on the cytoplasmic leaflet of cellular membranes (which include vesicular membranes), may help to stabilize the fusion pore before its transition to rapid dilation.

\section{Materials and Methods}

Cell preparation. Male Sprague Dawley rats (200-250 g) were killed in accordance with the standards of the Canadian Council on Animal Care. 
A

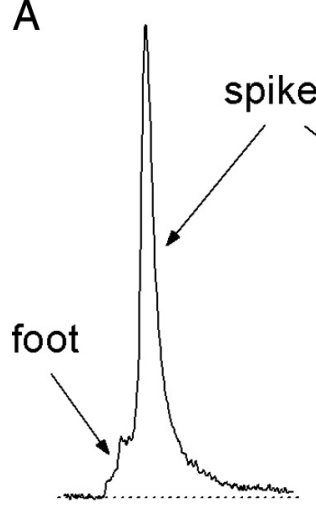

spike with a "foot"
B

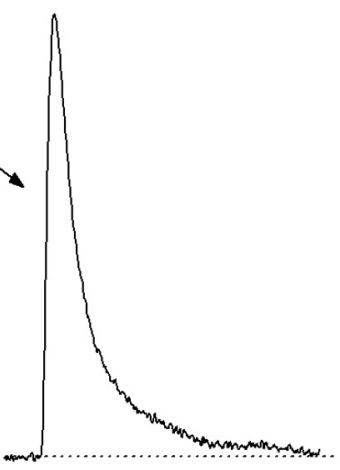

spike without a "foot"
C

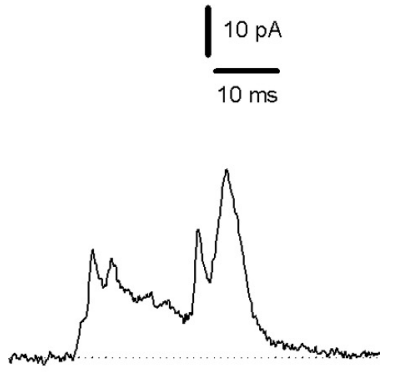

"stand-alone foot"

Figure 1. Different types of amperometric signals from rat chromaffin cells. Representative traces of spikes with $(\boldsymbol{A})$ or without (B) a prespike foot signal and a stand-alone foot signal ( $($ ).

A

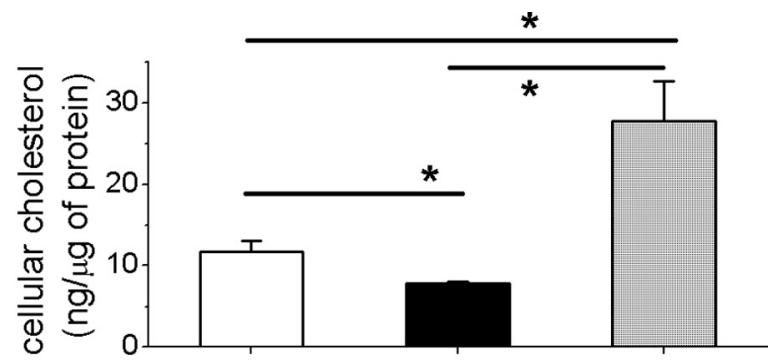

$\mathrm{B}$

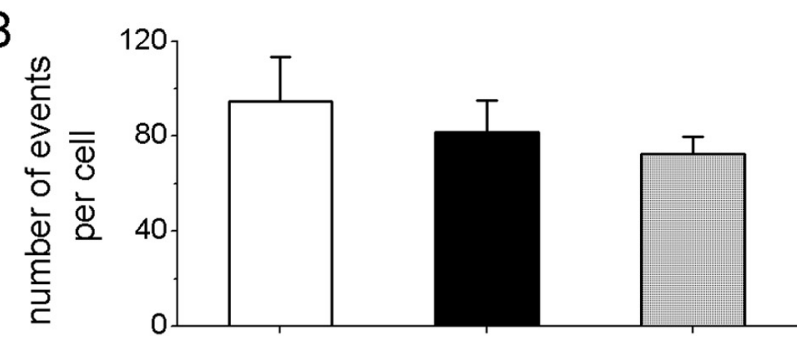

C

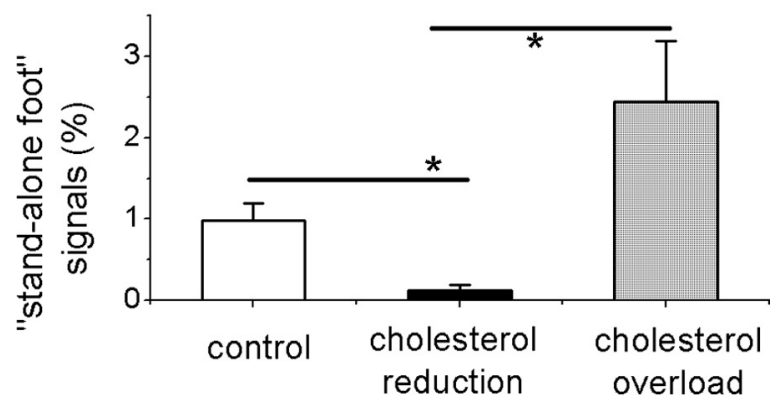

Figure 2. Perturbation of cellular cholesterol level did not affect the number of amperometric events recorded from individual cells but changed the proportion of stand-alone foot signals. $A$, The total cellular cholesterol level was reduced by $\sim 30 \%$ in cells treated with lovastatin and $\mathrm{M} \beta C D$ (cholesterol reduction) but increased by $\sim 2.4$-fold in cells treated with cholesterol (cholesterol overload). Data averaged from three experiments. $\boldsymbol{B}$, The number of events per cell (during the $5 \mathrm{~min}$ of whole-cell dialysis of $\mathrm{Ca}^{2+}$-buffered solution) was not affected by changes in cholesterol level. $C$, The proportion of stand-alone foot signals was decreased by cholesterol reduction but increased by cholesterol overload. In $\boldsymbol{B}$ and $\boldsymbol{C}$, the data were generated from 28 control cells (2664 events), 32 cells ( 2720 events) for cholesterol reduction, and 32 cells (2377 events) for cholesterol overload.

The adrenal medullae were dissociated enzymatically into single chromaffin cells as described previously (Xu and Tse, 1999; Xu et al., 2002, 2005). Cells were maintained in a defined medium [MEM supplemented with $1 \%(\mathrm{v} / \mathrm{v})$ insulin-transferrin-selenium-A, $50 \mathrm{U} / \mathrm{ml}$ penicillin $\mathrm{G}$, and 50 $\mu \mathrm{g} / \mathrm{ml}$ streptomycin). All culture materials were from Invitrogen. Recordings were performed at room temperature $\left(20-23^{\circ} \mathrm{C}\right)$ on cells maintained in culture for $1 \mathrm{~d}$.

Triggering of catecholamine release. Individual cells were voltage clamped in whole-cell mode at $-70 \mathrm{mV}$. Exocytosis was triggered via whole-cell dialysis of a $\mathrm{Ca}^{2+}$-buffered solution $\left(\left[\mathrm{Ca}^{2+}\right]\right.$ estimated to be $\sim 0.5 \mu \mathrm{M}$ at $22^{\circ} \mathrm{C}$ ) (Blinks et al., 1982) that contained the following (in mM): 40 KAsp, 40 HEPES, $20 \mathrm{KCl}, 20$ EGTA, $17 \mathrm{CaCl}_{2}, 2 \mathrm{Na}_{2} \mathrm{ATP}, 1 \mathrm{MgCl}_{2}$, and 0.1 GTP, $\mathrm{pH}$ 7.4. The standard bath solution contained the following (in $\mathrm{mm}$ ): $150 \mathrm{NaCl}, 10$ HEPES, 8 glucose, $2.5 \mathrm{KCl}, 2.5 \mathrm{CaCl}_{2}$, and 1 $\mathrm{MgCl}_{2}, \mathrm{pH}$ 7.4.

Perturbation and measurement of cellular cholesterol. The reagents to manipulate cholesterol (all from Sigma-Aldrich) included methyl- $\beta$ cyclodextran (M $\beta \mathrm{CD})$ (a cholesterol extractor), lovastatin (an inhibitor of cholesterol biosynthesis), and "soluble" cholesterol (with M $\beta C D$ as carrier). Chromaffin cells collected from several rats were divided into three groups (control, "cholesterol reduction," and "cholesterol overload"). To reduce cellular cholesterol, acutely isolated cells were cultured with lovastatin (5 $\mu \mathrm{M}$ ) overnight (12-16 h), and $\mathrm{M} \beta \mathrm{CD}$ (5 mM) was added to the medium for $1 \mathrm{~h}$ (immediately before the amperometry experiments). To increase cellular cholesterol, cells preincubated with lovastatin for 12-16 h were treated for $1 \mathrm{~h}$ with soluble cholesterol $(0.1 \mathrm{mg} / \mathrm{ml})$. Because $\mathrm{M} \beta C D$ is the carrier in the soluble cholesterol, the addition of soluble cholesterol to the lovastatin-treated cells allows us to eliminate possible non-cholesterolrelated effects of $\mathrm{M} \beta \mathrm{CD}$ as the major difference between the cholesterol reduction and cholesterol overload cells is the level of free cholesterol. Untreated cells with the same duration of culture and mock solution changes were used as controls. For experiments involving acute extraction of cholesterol from the cytosolic side, $10 \mathrm{~mm} \mathrm{M} \beta C D$ was included in the whole-cell pipette solution. For experiments involving acute perturbation of cholesterol from the extracellular side, $10 \mathrm{~mm} \mathrm{M} \beta \mathrm{CD}$ or 0.1 $\mathrm{mg} / \mathrm{ml}$ soluble cholesterol was added to the bath solution before the establishment of whole-cell configuration.

For measurement of cellular cholesterol, cultured chromaffin cells were harvested, and their amount of cholesterol and protein were quantified with the Amplex Red cholesterol assay kit (Invitrogen) and BCA protein assay kit (Thermo Fisher Scientific).

Amperometry and signal analysis. Carbon fiber (tip diameter of $\sim 7$ $\mu \mathrm{m})$ amperometry was used to monitor quantal release as described previously (Tang et al., 2007). Briefly, $+700 \mathrm{mV}$ was applied to the carbon fiber electrode, using a VA10 amplifier (NPI Electronics). The unfiltered output of the amplifier was low passed at $1 \mathrm{kHz}$ (Frequency Device). In each experiment, the carbon fiber electrode was positioned to touch the cell before the start of whole-cell dialysis. For all experiments, we collected data for $5 \mathrm{~min}$ after the start of whole-cell dialysis. All amperometric signals were digitized at $10 \mathrm{kHz}$ with a Digidata $1322 \mathrm{~A}$ running on pClamp 9.0 software (Molecular Devices) and analyzed with the Mini Analysis Program version 5.24 (Synaptosoft). Q was calculated from the time integral of individual amperometric events. We restricted our analysis to individual nonoverlapping amperometric signals using the same criteria as described in our previous studies (Tang et al., 2005, 2007): (1) the amplitude of amperometric events must be five times rms noise; (2) $50-90 \%$ rise time must be $<5 \mathrm{~ms}$; (3) the decay time must be $<40 \mathrm{~ms}$; and (4) the interval between the peaks of two adjacent amperometric events must be more than three times the decay time constant of the first amperometric event. Origin 7.5 (OriginLab) was used for plotting and statistical analysis. The two populations Student's $t$ test was used when comparing between mean values from control cells and cells with perturbed cholesterol levels (statistical $n$ indicates number of cells). Values of $p<0.05$ were considered statistically significant and were marked with asterisks in the figures. When comparing the distributions of each amperometric parameter over the entire range of $\mathrm{Q}^{1 / 3}$ for a pair of ex- 
perimental conditions (see Figs. 3-6), the Kolmogorov-Smirnov statistics (statistical $n$ indicates number of events) included in the Mini Analysis Program was used.

\section{Results}

Perturbation of cellular cholesterol level selectively affected the prespike foot signal

To bypass any possible effect of cholesterol perturbation on ion channels and their colocalization with sites of exocytosis, we triggered quantal catecholamine release from rat chromaffin cells via whole-cell dialysis of a $\mathrm{Ca}^{2+}$-buffered solution $\left(\left[\mathrm{Ca}^{2+}\right]_{\mathrm{i}}\right.$ of $\left.\sim 0.5 \mu \mathrm{M}\right)$. As shown in Figure 1 , the modest $\left[\mathrm{Ca}^{2+}\right]_{\mathrm{i}}$ elevation triggered three types of amperometric signals that were similar to those described in our previous studies in which high extracellular $\left[\mathrm{K}^{+}\right]$was used to trigger quantal release (Tang et al., 2005, 2007). The majority $(\sim 3 / 4)$ of the signals comprised events with a main spike that was preceded by a foot signal (Fig. $1 A$ ), which represents a fusion pore that initially stayed at a modest size for a measurable duration (typically several milliseconds) before the onset of rapid dilation. A smaller fraction $(\sim 1 / 4)$ of the signals were spikes without a detectable "prespike foot" signal (Fig. $1 B$ ), which reflects a fusion pore that either dilated rapidly with no detectable delay or was initially too small to support a detectable rate of catecholamine release until the onset of rapid dilation (Gong et al., 2007). A much smaller fraction $(\sim 1 \%)$ of the signals comprised the "standalone foot" signals (Fig. 1C), which represents a flickering fusion pore that might not proceed to the stage of rapid dilation.

To reduce cellular cholesterol, we incubated chromaffin cells with $5 \mu \mathrm{M}$ lovastatin (an inhibitor of the 3-hydroxy-3methyglutaryl coenzyme A reductase) overnight (12-16 h), followed by a $1 \mathrm{~h}$ treatment with the cholesterol extractor $\mathrm{M} \beta \mathrm{CD}$ ( 5 $\mathrm{mm})$. As shown in Figure $2 A$, this procedure reduced the mean cellular cholesterol (normalized to total cellular protein) by $\sim 30 \%$ (cholesterol reduction). When cells similarly preincubated with lovastatin were treated for $1 \mathrm{~h}$ with soluble cholesterol $(0.1 \mathrm{mg} / \mathrm{ml})$, the cellular cholesterol was increased to $\sim 2.4$-fold of control (Fig. 2A). Thus, this procedure not only reversed the cholesterol-lowering effect of lovastatin but overload the cells with cholesterol (cholesterol overload). We compared the amperometric signals collected from 28 control cells (2664 events), 32 cells with cholesterol reduction (2720 events), and 32 cells with cholesterol overload (2377 events). We found that changes in cellular cholesterol level did not affect the mean number of amperometric events collected from individual cells over the duration of $5 \mathrm{~min}$ of whole-cell dialysis of the $\mathrm{Ca}^{2+}$-buffered solution (Fig. 2B). However, the lowering of cellular cholesterol reduced the proportion of stand-alone foot signal (to $\sim 1 / 8$ that of control), whereas elevation of cholesterol had the opposite effect (increased to $\sim 2.5$-fold of controls) (Fig. $2 C$ ). In contrast, the time integral of individual amperometric events (i.e., Q) was unaffected by the changes in cellular cholesterol. As shown in Figure
Table 1. Reduction or elevation of cellular cholesterol had no significant effect on the spike kinetics

\begin{tabular}{llll}
\hline Treatment & Spike amplitude & Spike half-width & $\begin{array}{l}\text { Spike decay } \\
\text { time constant }\end{array}$ \\
\hline $\begin{array}{l}\text { Control ( } n=28 \text { cells; } \\
\quad 2664 \text { events) }\end{array}$ & $26.0 \pm 4.1 \mathrm{pA}$ & $5.5 \pm 0.3 \mathrm{~ms}$ & $7.4 \pm 0.4 \mathrm{~ms}$ \\
$\begin{array}{c}\text { Cholesterol reduction } \\
(n=32 \text { cells; } 2720 \text { events) }\end{array}$ & $24.6 \pm 3.2 \mathrm{pA}$ & $5.9 \pm 0.3 \mathrm{~ms}$ & $7.9 \pm 0.4 \mathrm{~ms}$ \\
$\begin{array}{c}\text { Cholesterol overload } \\
(n=32 \text { cells; } 2377 \text { events })\end{array}$ & $26.5 \pm 3.1 \mathrm{pA}$ & $5.6 \pm 0.3 \mathrm{~ms}$ & $7.5 \pm 0.4 \mathrm{~ms}$ \\
\hline
\end{tabular}

Values shown were the mean $\pm S E$ of specific kinetic parameters averaged from spikes of individual cells in a treatment group.

$3 A$, there was no difference in the mean value (Fig. 3Ai) or the distribution of $\mathrm{Q}^{1 / 3}$ (Fig. 3Aii) between the control cells and cells with perturbed cholesterol levels. Figure $3 B$ shows that changes in cellular cholesterol level also did not affect the mean proportion of spikes with a prespike foot signal recorded from individual cells (Fig. 3Bi) or the percentage of such signals at different values of $\mathrm{Q}^{1 / 3}$ (Fig. 3Bii).

In our previous study, when amperometric signals from rat chromaffin cells were compared at matched values in Q, the spike kinetics for events with a prespike foot tended to be more rapid (e.g., with smaller half-width) than those without a detectable prespike foot signal (Tang et al., 2007). Therefore, when examining the effect of cholesterol on spike kinetics, we either analyzed the events with or without a prespike foot signal separately or combined both types of signals in our analysis. Each kinetic parameter of the spike was analyzed with two methods. In the first 

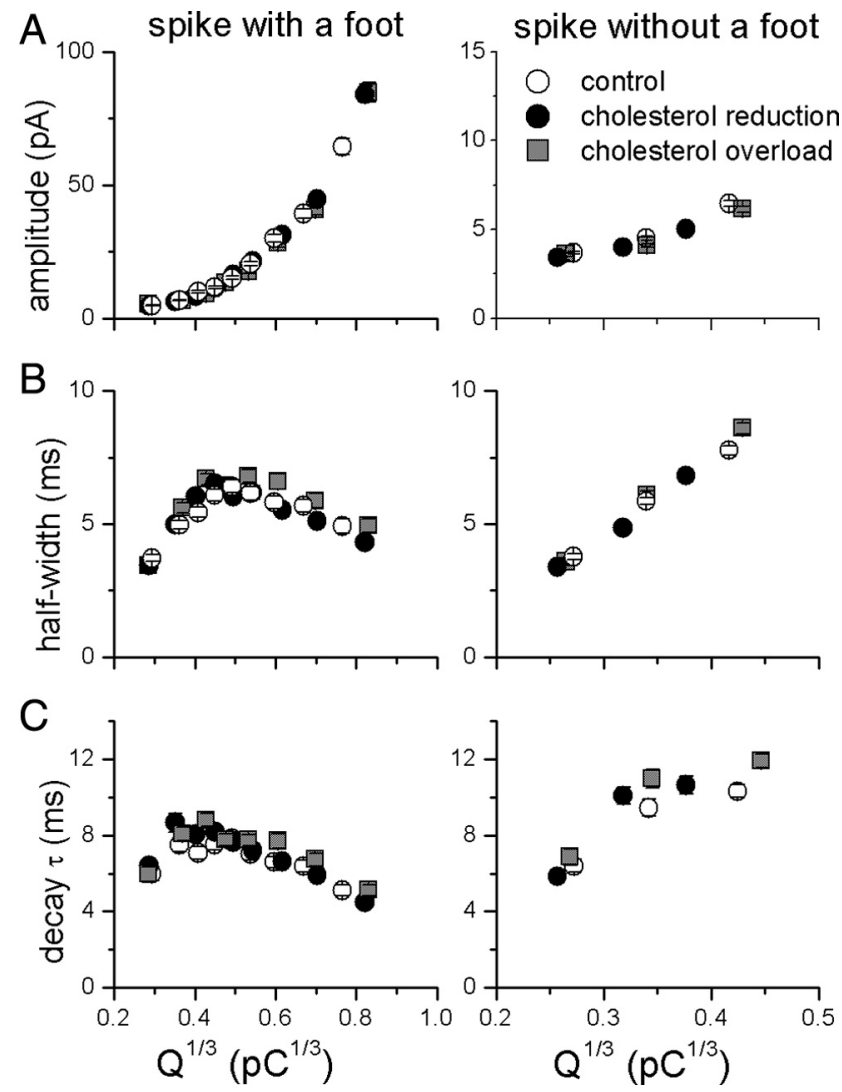

Figure 4. Changes in cellular cholesterol level did not affect the different kinetic parameters of the main spike. Plots of the amplitude $(\boldsymbol{A})$, half-width $(\boldsymbol{B})$, and decay $\tau(\boldsymbol{C})$ of the main spike at different values of $Q^{1 / 3}$ for events with or without a prespike foot signal. Each data point was the mean \pm SE from 200 events. Kolmogorov-Smirnov tests showed that the distributions for each perturbation of cholesterol in $\boldsymbol{A}-\boldsymbol{C}$ were not significantly different from the control.

method, we calculated the mean values of a parameter (e.g., spike amplitude) from all the spikes collected from individual cells and then averaged these values from all the cells in the same treatment group (i.e., average of the cell mean values) (Table 1). This analysis minimized sampling bias that might be introduced by any overrepresentation of individual cells that contributed an exceptionally large number of signals (Mosharov and Sulzer, 2005). With this analysis, we found no significant differences in spike kinetics among the treatment groups (cholesterol reduction and cholesterol overload) and the control cells (Table 1). In the second method, the events for each treatment were grouped according to their quantal size, and the specific kinetic parameters were plotted as a function of $\mathrm{Q}^{1 / 3}$ (Fig. 4). This analysis allows us to examine whether cholesterol has any selective effect on spike kinetics at certain ranges of $\mathrm{Q}^{1 / 3}$. As shown in Figure 4, for the main spike of events with or without a prespike foot signal, changes in cellular cholesterol did not affect the peak amplitude (Fig. $4 A$ ), half-width (Fig. $4 B$ ), and decay $\tau$ (Fig. $4 C$ ) at any range of $\mathrm{Q}^{1 / 3}$.

In contrast, we found that changes in cellular cholesterol clearly affected the duration of the prespike foot signals (Fig. 5). Cholesterol reduction caused a significant decrease in the average value of the mean foot duration from individual cells, whereas cholesterol overload had the opposite effect (Fig. 5Ai). As shown in Figure 5Aii, the two manipulations of cholesterol resulted in opposite changes in foot duration for events over a wide range of $\mathrm{Q}^{1 / 3}$. Although cholesterol reduction and overload also had op-
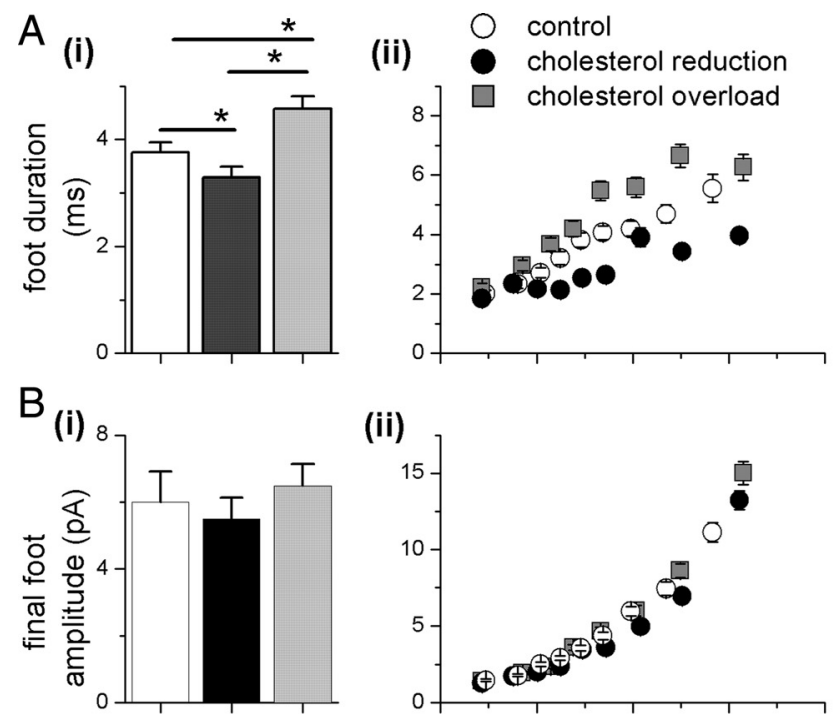

(ii)
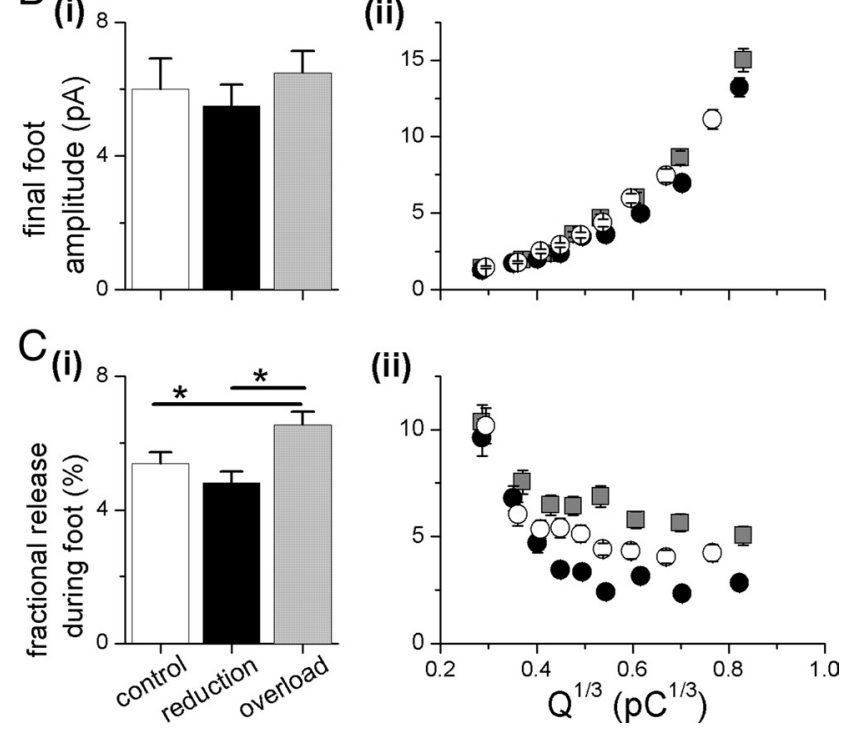

Figure 5. Perturbations of cellular cholesterol level affected the duration of the semi-stable fusion pore and the fractional release during the prespike foot signal. $A$, Cholesterol reduction decreased the foot duration, and cholesterol overload had opposite effect. $\boldsymbol{B}$, Changes in cholesterol level had no significant effect on the final amplitude of the prespike foot signal. $\boldsymbol{C}$, The changes in the foot duration in turn led to corresponding changes in the fractional release during foot. Ci, Plots of the average of the cell mean values. Cii, Plots of specific kinetic parameter at different values of $Q^{1 / 3}$. Each data point was the mean \pm SE from 200 events. Kolmogorov-Smirnov tests showed that the distributions for each perturbation of cholesterol in Aii and Cii, but not Bii, was significantly different from the control.

posite trends on the final amplitude of the prespike foot signal, such trends were statistically insignificant (Fig. 5B). The proportional changes in the fractional release during the prespike foot signal associated with the perturbations of cellular cholesterol were similar to those in the foot duration for both the mean cellular value (Fig. 5Ai,Ci) and at any particular range of $\mathrm{Q}_{1 / 3}^{1 / 3 i g}$. $5 A$ ii,Cii). Note that the effect of cholesterol overload on foot duration was more robust than cholesterol reduction $(\sim 22 \%$ increase in foot duration vs $\sim 13 \%$ reduction when compared with control) (Fig. 5Ai). This probably contributed to the significant increase in the average value of the mean fractional release during the prespike foot signal in cholesterol overload cells when compared with the control cells (Fig. 5Ci), whereas cholesterol reduction caused no significant change in a similar comparison. Nevertheless, the average value of the mean fractional release during the foot signal in cells with cholesterol reduction was significantly smaller when compared with the cholesterol overload cells (Fig. 5Ci).

Acute extraction of cholesterol from the cytosol affected the foot signals

In the above experiments, our procedure to reduce cellular cholesterol is expected to perturb multiple pools of cellular choles- 

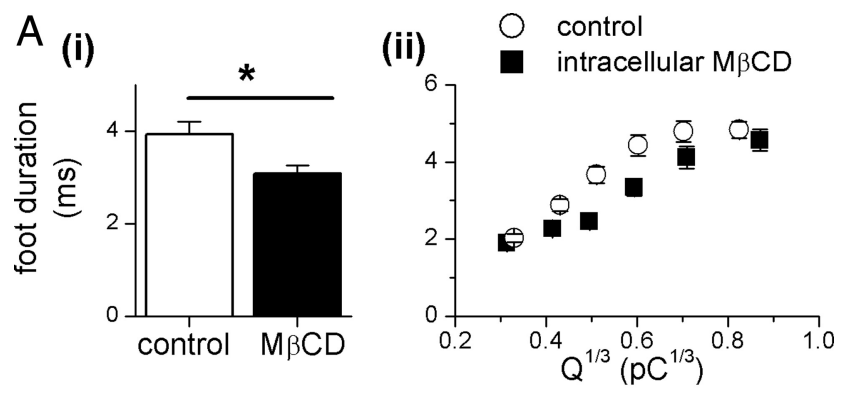

$\mathrm{B}$ (i)

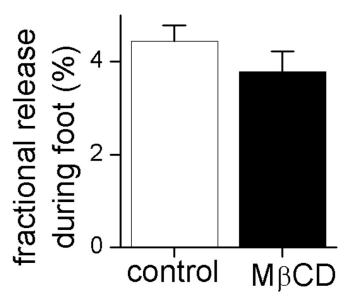

(ii)
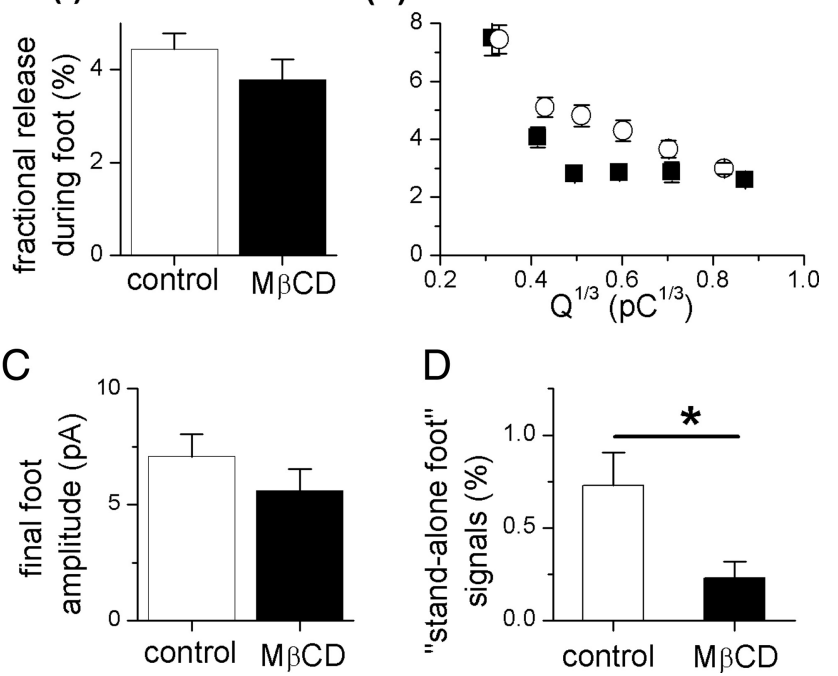

Figure 6. Effects of acute cholesterol extraction from the cytosolic compartments. Ai, The average value of the mean foot duration from individual cells dialyzed with $M \beta C D$ (17 cells; 2632 events) was significantly smaller than the controls ( 20 cells; 2636 events). Aii, Cytoplasmic extraction of cholesterol (intracellular $M \beta C D$ ) reduced the duration of the prespike foot signal for events in a wide range of $Q^{1 / 3}$. B , The reduction in foot duration in turn decreased the fractional release during the foot in a range of $Q^{1 / 3}$ (ii), but the reduction in the average value of the mean fractional release from individual cells was not statistically significant (i). C, IntracelIular $M \beta C D$ did not cause any significant change in the average value of the mean final foot amplitude from individual cells. $D$, The proportion of stand-alone foot signals was significantly reduced in cells dialyzed with $M \beta C D$. Each data point in Aii and Bii was the mean \pm SE from 200 events. Kolmogorov-Smirnov tests showed that the distributions for the treatment with $\mathrm{M} \beta C D$ in Aii and Bii were significantly different from the control.

terol (Rothblat et al., 1999). For example, the M $\beta C D$ treatment is expected to extract the cholesterol on the extracellular side of the plasma membrane most efficiently [with a half-time in the range of seconds at room temperature (Steck et al., 2002)]. Conversely, treatment with lovastatin is expected to decrease cholesterol synthesis and hence be more efficient in reducing the pool of cellular cholesterol that is recently synthesized (e.g., at the cytosolic side of the endoplasmic reticulum). Moreover, the half-time for the trans-bilayer movement of cholesterol might be on the order of 1 s (Steck et al., 2002), whereas the half-time for transport of cholesterol from the endoplasmic reticulum to the plasma membrane might range from $10 \mathrm{~min}$ to $1 \mathrm{~h}$ (Liscum and Dahl, 1992). Thus, it is likely that our procedures also affected other pools of cholesterol, some of which were probably located at membranes in direct contact with the cytosol. Such pools include the cholesterol on the cytosolic side of the plasma membrane and the membrane of various intracellular organelles [including the secretory vesicles, in which the molecular ratio of cholesterol to phospholipids was reported to be $\sim 0.5$ (Churchward et al., 2005)].
To examine whether the cholesterol at the cytosolic side of various membranes or at the extracellular side of the plasma membrane played a more prominent role in regulating the kinetics of fusion pore, we compared the effects of acute $(\sim 5 \mathrm{~min})$ extraction of cholesterol from the cytosolic side with those of acute extracellular extraction. Figure $6 A i$ shows that, for cells recorded with $10 \mathrm{mM} \mathrm{M} \beta \mathrm{CD}$ in the cytosol (applied via wholecell dialysis; 17 cells and 2632 events), there was a significant decrease (by $\sim 21 \%$ ) in the average value of the mean foot duration from individual cells when compared with the controls $(20$ cells; 2636 events). As shown in Figure 6Aii, intracellular M $\beta C D$ reduced the duration of the prespike foot signal at a large range of $\mathrm{Q}\left(\mathrm{Q}^{1 / 3}\right.$ of $\left.0.4-0.7 \mathrm{pC}^{1 / 3}\right)$. This effect was accompanied by a comparable reduction in the fractional release during the prespike foot signal in the same range of Q (Fig. 6 Bii), although the change in the average value of the mean fractional release from individual cells was not statistically significant (Fig. $6 \mathrm{Bi}$ ). Figure $6 C$ shows that the final amplitude of the prespike foot signal also tended to be smaller with intracellular dialysis of $\mathrm{M} \beta \mathrm{CD}$, although this effect was also statistically insignificant. In contrast, intracellular $\mathrm{M} \beta \mathrm{CD}$ caused a significant reduction in the proportion of stand-alone foot signals (Fig. 6D). Conversely, acute extracellular application of $10 \mathrm{~mm} \mathrm{M} \beta C D$ (20 cells; 1712 events) did not reduce the duration of the prespike foot signal, the fractional release during the prespike foot, or the proportion of stand-alone foot signal when compared with the control cells (21 cells; 2281 events) (Table 2 ). Thus, only the acute extraction of cholesterol from the cytosolic side mimicked the effects of the lowering of overall cellular cholesterol (combined treatment with lovastatin and $\mathrm{M} \beta \mathrm{CD}$ ) on both types of foot signals (Figs. 2C, 5A).

We also examined the effect of acute application of soluble cholesterol. However, the inclusion of soluble cholesterol in the whole-cell pipette hindered the formation of gigaseal. Therefore, we could only acutely apply soluble cholesterol from the extracellular side. In a study of PC12 cells, acute application of cholesterol was reported to evoke spontaneous release (Zhang et al., 2009). In contrast, our carbon fiber electrode did not detect any amperometric signal from chromaffin cells with extracellular application of soluble cholesterol. Amperometric signals were detected only after the establishment of whole-cell configuration when the $\mathrm{Ca}^{2+}$-buffered solution was dialyzed into the cell. As summarized in Table 3 , the addition of soluble cholesterol $(0.1$ $\mathrm{mg} / \mathrm{ml} ; 15$ cells; 721 events) to the bath solution did not have any significant effect on the kinetics of the prespike foot signal or the proportion of stand-alone foot signals when compared with the control cells ( 12 cells; 789 events). This result suggests that cholesterol overload from the extracellular side of the plasma membrane could not be rapidly (within $\sim 5 \mathrm{~min}$ ) delivered to the relevant sites to influence fusion pore kinetics. Our overall findings from all of the acute perturbation experiments suggest that the cholesterol molecules that contribute to the regulation of the foot signals can be extracted rapidly from the cytosolic side and cannot be oversupplied rapidly from the extracellular side of the plasma membrane.

Our previous results have shown that the perturbation of cellular cholesterol did not affect the mean number of amperometric events per cell (Fig. $2 B$ ), the $\mathrm{Q}^{1 / 3}$ distribution (Fig. 3A), the proportion of spike signals preceded by a foot (Fig. $3 B$ ), and the kinetics of the spike signals (Table 1, Fig. 4). Similarly, the above parameters were not affected by acute cholesterol extraction (intracellular or extracellular $\mathrm{M} \beta \mathrm{CD}$ ) or acute cholesterol overload (data not shown). 
Table 2. Acute application of extracellular $M \beta C D$ had no significant effect on the kinetics of the prespike foot signal or the proportion of stand-alone foot signals

\begin{tabular}{lllr}
\hline Treatment & Foot duration & Foot amplitude & Fractional release during foot \\
\hline Control ( $n=21$ cells; 2281 events $)$ & $4.1 \pm 0.3 \mathrm{~ms}$ & $11.9 \pm 0.9 \mathrm{pA}$ & $6.0 \pm 0.4 \%$ \\
Extracellular M $\beta C D(n=20$ cells; 1712 events $)$ & $4.9 \pm 0.6 \mathrm{~ms}$ & $10.1 \pm 1.4 \mathrm{pA}$ & $6.1 \pm 0.5 \%$ \\
\hline
\end{tabular}

Values shown were the mean \pm SE of specific kinetic parameters averaged from the prespike foot signals of individual cells and the proportion of stand-alone foot signals in a treatment group.

Table 3. Acute extracellular application of cholesterol had no significant effect on the kinetics of the prespike foot signal or the proportion of stand-alone foot signals

\begin{tabular}{llll}
\hline Treatment & Foot duration & Foot amplitude & Fractional release during foot \\
\hline Control ( $n=12$ cells; 789 events) & $4.6 \pm 0.6 \mathrm{~ms}$ & $13.6 \pm 3.0 \mathrm{pA}$ & $7.9 \pm 0.9 \%$ \\
Extracellular cholesterol ( $n=15$ cells; 721 events) & $3.8 \pm 0.4 \mathrm{~ms}$ & $11.6 \pm 2.0 \mathrm{pA}$ & $6.6 \pm 0.8 \%$ \\
\hline
\end{tabular}

Values shown were the mean \pm SE of specific kinetic parameters averaged from the prespike foot signals of individual cells and the proportion of stand-alone foot signals in a treatment group.

I cholesterol intercalated between lipids or proteins $\nabla$ inverted-cone shaped lipids

$\triangle$ cone shaped lipids

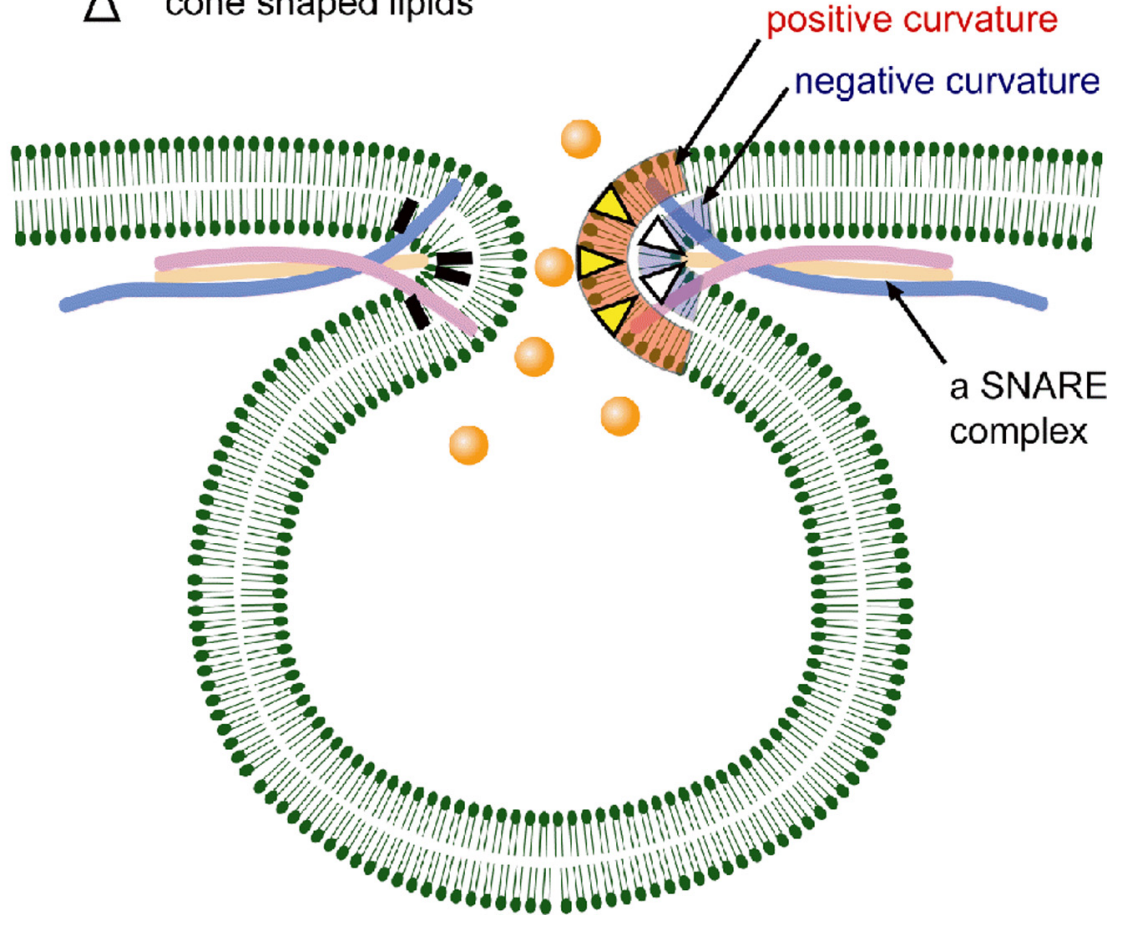

Figure 7. A model of the semi-stable fusion pore before the onset of rapid dilation. The narrow waistline of such a fusion pore is probably constrained by a ring of SNARE complexes (two are depicted) and associated proteins, and the lipid membrane must make a very sharp hairpin turn there. The hairpin turn of the fused cytoplasmic leaflets must have very negative curvature (blue semicircle) that can be promoted by cone-shaped lipids or by cholesterol molecules intercalating in the hydrophobic domain of phospholipids. In contrast, the hairpin turn involving the luminal leaflet of the vesicular membrane and the extracellular leaflet of the plasma membrane must have positive curvature (red semicircle) that can be promoted by inverted-cone shaped lipids. Molecules of the vesicular cargo are depicted as spheres.

\section{Discussion}

Influence of cholesterol on the persistence of the semi-stable fusion pore

Our study shows that extraction of cellular cholesterol reduced the proportion of stand-alone foot signals (Fig. 2C) and shortened the prespike foot signals (Fig. 5). In cell-attached amperometry, the time course of well resolved prespike foot signals has been shown to be closely correlated with fusion pore conductance (Gong et al., 2007). Thus, our results suggest that the lowering of cellular cholesterol reduced the persistence of the fusion pore of the LDCGs before the onset of rapid dilation. Conversely, elevation of cellular cholesterol level increased the persistence of the semi-stable fusion pores (Figs. 2C, 5). Changes in the prespike foot signals have been associated with elevations of cellular cAMP level in rat chromaffin cells (Tang et al., 2007), overexpression of certain synaptotagmin isoforms in PC12 cells (Wang et al., 2001), inhibition of actin polymerization in bovine chromaffin cells (Berberian et al., 2009), and cholesterol perturbation in PC12 cells (Zhang et al., 2009). In these studies, however, the changes in foot signals were accompanied by changes in quantal size and/or the kinetics of the main spike. In contrast, in our study, the effects of cholesterol perturbations on the prespike foot signals were not accompanied by any change in other amperometric parameters (Figs. 3, 4). A selective reduction in the duration of the prespike foot signal has also been reported in SNAP-25 (synaptosome-associated protein of $25 \mathrm{kDa}$ )-deficient chromaffin cells (Sørensen et al., 2003). The predominant effect of cholesterol perturbation on the prespike foot signal suggests that cholesterol may not have any major influence on the most rapid phase of catecholamine release during and after the rapid dilation of the fusion pore.

Our results contrast previous reports on HA-mediated fusion studies in which the addition of cholesterol prominently promoted membrane fusion and fusion pore expansion (Razinkov et al., 1998; Razinkov and Cohen, 2000; Biswas et al., 2008). The discrepancy between the HAmediated fusion and our study on chromaffin LDCGs may be attributable to fundamental differences between the two mechanisms of fusion. For example, the undilated fusion pore in the HA-mediated fusion may be more "lipidic" than the one for exocytosis of LDCGs (Jackson and Chapman, 2008).

Influence of cholesterol on spontaneous release

Cholesterol extraction was reported to augment "spontaneous" exocytosis of synaptic vesicles from the crayfish neuromuscular junction (Zamir and Charlton, 2006) and hippocampal neurons (Wasser et al., 2007). In contrast, acute cholesterol addition was reported to trigger spontaneous release in PC12 cells (Zhang et al., 2009). We did not detect any amperometric signal from chro- 
maffin cells before the onset of intracellular dialysis of the $\mathrm{Ca}^{2+}$ buffered solution in control condition, and none of our manipulations of cellular cholesterol level evoked any spontaneous amperometric signal. It is not clear whether the discrepancy was attributable to differences in cell types or the types of granules. In pituitary lactotrophs, spontaneous release was associated with a small fusion pore opening that was too narrow for the discharge of peptide hormones (Vardjan et al., 2007). A similar mechanism may account for the rare occurrences of spontaneous amperometric signal in chromaffin cells.

\section{The persistence of the semi-stable fusion pore is regulated by cholesterol molecules at the cytosolic leaflets}

We found that acute extraction of cholesterol from the cytosolic, but not the extracellular, compartments shortened the prespike foot signal and reduced the proportion of stand-alone foot signals (Fig. 6). This finding indicates that the cholesterol molecules in the cytosolic leaflet of the plasma membrane or vesicular membrane are more critical for increasing the persistence of the semistable fusion pore. The ability of intracellular $\mathrm{M} \beta \mathrm{CD}$ to rapidly extract cholesterol at the cytosolic leaflets of the fusion pore is consistent with the notion that cholesterol in the cytosolic leaflet have a higher tendency to escape to an aqueous acceptor such as M $\beta C D$ (Lange and Steck, 2008). We found that acute cholesterol overload from the extracellular side also failed to affect the two types of foot signals. Although cholesterol can flip across the plasma membrane rapidly, it is conceivable that chromaffin cells can initially overcome excessive cholesterol accumulation via stimulation of cholesterol efflux or intracellular esterification of cholesterol (Ikonen, 2006) and that a longer exposure to exogenous cholesterol (e.g., $1 \mathrm{~h}$ as in our experiment) is needed for sufficient cholesterol accumulation to occur at the relevant sites.

\section{Possible mechanisms underlying the influence of cholesterol on fusion pore}

It is generally accepted that the overall shape of a fusion pore for exocytosis just before the onset of dilation resembles the waistline of an hourglass (Jackson and Chapman, 2006). The narrowest part of such a fusion pore is probably constrained by a ring of SNARE complexes (Fig. 7). When such a fusion pore just starts to dilate, at least one pair of neighboring SNARE complexes must separate and allow at least a sliver of lipid molecules to insinuate between them. This sliver of lipid molecules must negotiate a sharp hairpin turn in which the two leaflets of the lipid membrane must have opposite curvatures: the one facing the lumen of the pore and continuous with extracellular side must be positive (i.e., the polar head groups of the typical phospholipids are spaced farther apart than those in a planar lipid leaflet), whereas the cytoplasmic leaflet must be negative (i.e., the hydrophopic tails of the typical phospholipids are spaced farther apart than those in a planar lipid leaflet).

Three physical properties of cholesterol molecules in (proteinfree) lipid membranes (Chen and Rand, 1997) may contribute to stabilize the structure depicted in Figure 7. First, cholesterol can increase the overall viscosity of the lipid membrane; this property is expected to impede the flux of lipid molecules that are necessary for dilating the waistline of the fusion pore. Second, cholesterol can slightly increase the bending modulus (i.e., stiffness) of a lipid monolayer. Third, cholesterol can decrease the intrinsic (spontaneous) radius of a lipid monolayer in a way similar to cone-shaped lipids. If there is indeed more cholesterol in the cytosolic leaflet (compared with the leaflet facing the lumen of the fusion pore), it will confer a negative curvature in that leaflet in a way similar to a cone-shaped lipid, Together, these three properties will make it energetically favorable to form and to maintain the narrow waistline of the fusion pore. The ability of cholesterol in conferring negative curvature suggests that a decrease in cholesterol on the cytoplasmic leaflets of the fusion pore may lower the overall probability of successful fusion. Consistent with this, cholesterol extraction reduced the fraction of sea urchin cortical vesicles that could be triggered to undergo $\mathrm{Ca}^{2+}$-dependent exocytosis or vesicle-vesicle fusion, and this effect could be reversed by the addition of lipids with comparable negative curvature to cholesterol (Churchward et al., 2005, 2008). A reduction of the "evoked" release (triggered by hypertonic sucrose) was also observed in hippocampal cultures treated with $\mathrm{M} \beta \mathrm{CD}$ and in hippocampal cultures from Niemann-Pick type C1-deficient mice defective in intracellular cholesterol trafficking (Wasser et al., 2007). In our study, however, the perturbations of cholesterol did not alter the total number of amperometric events recorded (Fig. 2 ). It is probable that the extent of cholesterol extraction at the relevant sites in our experiments was modest when compared with the isolated urchin cortical vesicle preparation or the synapses of the hippocampal neurons, such that sufficient cholesterol remains in the chromaffin cells for proper fusion pore assembly and opening. Alternatively, in addition to cholesterol molecules, the cytoplasmic leaflet of the plasma or vesicular membrane of chromaffin cells may have other lipids that can support the negative curvature of the fusion pore.

In addition to the three physical properties of cholesterol mentioned above, it is also possible that cholesterol influences fusion pore kinetics via cholesterol-protein interactions. For example, the vesicular SNARE synaptobrevin was recently reported to interact directly with a lipid, sphingosine, at the synaptobrevin/phospholipid interface to enhance exocytosis (Darios et al., 2009). If cholesterol also has high affinity for certain transmembrane domains of the neighboring SNARE complexes (Jeremic et al., 2006), then an oversupply of cholesterol at the cytosolic leaflet of the fusion pore will increase the stability of the fusion pore. Cholesterol extraction has also been reported to reduce the level of phosphatidylinositol 4,5-bisphosphate in plasma membrane (Kwik et al., 2003), which in turn led to a disorganization of the filamentous actin (F-actin) network (Hao and Bogan, 2009). The disruption of the actin network with cytochalasin $\mathrm{D}$ has been shown to lengthen the prespike foot signal and the spike halfwidth in bovine chromaffin cells (Berberian et al., 2009). Note, however, that cholesterol extraction caused a shortening of the prespike foot signal in our study, and there was no change in spike half-width. Thus, it is unlikely that the effect of cholesterol in our study is attributable to secondary changes in the F-actin network. In light of the many potential interactions among cholesterol, membrane phospholipids, and SNARE proteins (and their associated proteins), the challenge of future studies is to assess their relative contributions in the context of exocytosis.

\section{References}

Berberian K, Torres AJ, Fang Q, Kisler K, Lindau M (2009) F-actin and myosin II accelerate catecholamine release from chromaffin granules. J Neurosci 29:863-870.

Biswas S, Yin SR, Blank PS, Zimmerberg J (2008) Cholesterol promotes hemifusion and pore widening in membrane fusion induced by influenza hemagglutinin. J Gen Physiol 131:503-513.

Blinks JR, Wier WG, Hess P, Prendergast FG (1982) Measurement of $\mathrm{Ca}^{2+}$ concentrations in living cells. Prog Biophys Mol Biol 40:1-114.

Chen Z, Rand RP (1997) The influence of cholesterol on phospholipid membrane curvature and bending elasticity. Biophys J 73:267-276.

Churchward MA, Rogasevskaia T, Höfgen J, Bau J, Coorssen JR (2005) 
Cholesterol facilitates the native mechanism of $\mathrm{Ca}^{2+}$-triggered membrane fusion. J Cell Sci 118:4833-4848.

Churchward MA, Rogasevskaia T, Brandman DM, Khosravani H, Nava P, Atkinson JK, Coorssen JR (2008) Specific lipids supply critical intrinsic negative curvature: an essential component of native $\mathrm{Ca}^{2+}$-triggered membrane fusion. Biophys J 94:3976-3986.

Darios F, Wasser C, Shakirzyanova A, Giniatullin A, Goodman K, MunozBravo JL, Raingo J, Jorgacevski J, Kreft M, Zorec R, Rosa JM, Gandia L, Gutiérrez LM, Binz T, Giniatullin R, Kavalali ET, Davletov B (2009) Sphingosine facilitates SNARE complex assembly and activates synaptic vesicle exocytosis. Neuron 62:683-694.

Elhamdani A, Palfrey HC, Artalejo CR (2001) Quantal size is dependent on stimulation frequency and calcium entry in calf chromaffin cells. Neuron 31:819-830.

Elhamdani A, Azizi F, Artalejo CR (2006) Double patch clamp reveals that transient fusion (kiss-and-run) is a major mechanism of secretion in calf adrenal chromaffin cells: high calcium shifts the mechanism from kissand-run to complete fusion. J Neurosci 26:3030-3036.

Gong LW, de Toledo GA, Lindau M (2007) Exocytotic catecholamine release is not associated with cation flux through channels in the vesicle membrane but $\mathrm{Na}^{+}$influx through the fusion pore. Nat Cell Biol 9:915-922.

Hao M, Bogan JS (2009) Cholesterol regulates glucose-stimulated insulin secretion through phosphatidylinositol 4,5-bisphosphate. J Biol Chem 284:29489-29498.

Ikonen E (2006) Mechanisms for cellular cholesterol transport: defects and human disease. Physiol Rev 86:1237-1261.

Jackson MB, Chapman ER (2006) Fusion pores and fusion machines in $\mathrm{Ca}^{2+}$-triggered exocytosis. Annu Rev Biophys Biomol Struct 35:135-160.

Jackson MB, Chapman ER (2008) The fusion pores of $\mathrm{Ca}^{2+}$-triggered exocytosis. Nat Struct Mol Biol 15:684-689.

Jeremic A, Jin Cho W, Jena BP (2006) Cholesterol is critical to the integrity of neuronal porosome/fusion pore. Ultramicroscopy 106:674-677.

Kwik J, Boyle S, Fooksman D, Margolis L, Sheetz MP, Edidin M (2003) Membrane cholesterol, lateral mobility, and the phosphatidylinositol 4,5bisphosphate-dependent organization of cell actin. Proc Natl Acad Sci U S A 100:13964-13969.

Lang T, Bruns D, Wenzel D, Riedel D, Holroyd P, Thiele C, Jahn R (2001) SNAREs are concentrated in cholesterol-dependent clusters that define docking and fusion sites for exocytosis. EMBO J 20:2202-2213.

Lange Y, Steck TL (2008) Cholesterol homeostasis and the escape tendency (activity) of plasma membrane cholesterol. Prog Lipid Res 47:319-332.

Liscum L, Dahl NK (1992) Intracellular cholesterol transport. J Lipid Res 33:1239-1254.

Mosharov EV, Sulzer D (2005) Analysis of exocytotic events recorded by amperometry. Nat Methods 2:651-658.

Ohara-Imaizumi M, Nishiwaki C, Kikuta T, Kumakura K, Nakamichi Y, Nagamatsu S (2004) Site of docking and fusion of insulin secretory granules in live MIN6 beta cells analyzed by TAT-conjugated antisyntaxin 1 antibody and total internal reflection fluorescence microscopy. J Biol Chem 279:8403-8408.

Razinkov VI, Cohen FS (2000) Sterols and sphingolipids strongly affect the growth of fusion pores induced by the hemagglutinin of influenza virus. Biochemistry 39:13462-13468.

Razinkov VI, Melikyan GB, Epand RM, Epand RF, Cohen FS (1998) Effects of spontaneous bilayer curvature on influenza virus-mediated fusion pores. J Gen Physiol 112:409-422.

Rothblat GH, de la Llera-Moya M, Atger V, Kellner-Weibel G, Williams DL, Phillips MC (1999) Cell cholesterol efflux: integration of old and new observations provides new insights. J Lipid Res 40:781-796.

Salaün C, James DJ, Chamberlain LH (2004) Lipid rafts and the regulation of exocytosis. Traffic 5:255-264.
Sombers LA, Hanchar HJ, Colliver TL, Wittenberg N, Cans A, Arbault S, Amatore C, Ewing AG (2004) The effects of vesicular volume on secretion through the fusion pore in exocytotic release from PC12 cells. J Neurosci 24:303-309.

Sørensen JB, Nagy G, Varoqueaux F, Nehring RB, Brose N, Wilson MC, Neher E (2003) Differential control of the releasable vesicle pools by SNAP-25 splice variants and SNAP-23. Cell 114:75-86.

Steck TL, Ye J, Lange Y (2002) Probing red cell membrane cholesterol movement with cyclodextrin. Biophys J 83:2118-2125.

Tang KS, Tse A, Tse FW (2005) Differential regulation of multiple populations of granules in rat adrenal chromaffin cells by culture duration and cyclic AMP. J Neurochem 92:1126-1139.

Tang KS, Wang N, Tse A, Tse FW (2007) Influence of quantal size and cAMP on the kinetics of quantal catecholamine release from rat chromaffin cells. Biophys J 92:2735-2746.

Taverna E, Saba E, Rowe J, Francolini M, Clementi F, Rosa P (2004) Role of lipid microdomains in P/Q-type calcium channel (Cav2.1) clustering and function in presynaptic membranes. J Biol Chem 279:5127-5134.

Taverna E, Saba E, Linetti A, Longhi R, Jeromin A, Righi M, Clementi F, Rosa $P$ (2007) Localization of synaptic proteins involved in neurosecretion in different membrane microdomains. J Neurochem 100:664-677.

Vardjan N, Stenovec M, Jorgacevski J, Kreft M, Zorec R (2007) Subnanometer fusion pores in spontaneous exocytosis of peptidergic vesicles. J Neurosci 27:4737-4746.

Vikman J, Jimenez-Feltström J, Nyman P, Thelin J, Eliasson L (2009) Insulin secretion is highly sensitive to desorption of plasma membrane cholesterol. FASEB J 23:58-67.

Wang CT, Grishanin R, Earles CA, Chang PY, Martin TF, Chapman ER, Jackson MB (2001) Synaptotagmin modulation of fusion pore kinetics in regulated exocytosis of dense-core vesicles. Science 294:1111-1115.

Wasser CR, Ertunc M, Liu X, Kavalali ET (2007) Cholesterol-dependent balance between evoked and spontaneous synaptic vesicle recycling. J Physiol 579:413-429.

Xia F, Gao X, Kwan E, Lam PP, Chan L, Sy K, Sheu L, Wheeler MB, Gaisano HY, Tsushima RG (2004) Disruption of pancreatic beta-cell lipid rafts modifies Kv2.1 channel gating and insulin exocytosis. J Biol Chem 279:24685-24691.

Xia F, Leung YM, Gaisano G, Gao X, Chen Y, Fox JE, Bhattacharjee A, Wheeler MB, Gaisano HY, Tsushima RG (2007) Targeting of voltagegated $\mathrm{K}^{+}$and $\mathrm{Ca}^{2+}$ channels and soluble N-ethylmaleimide-sensitive factor attachment protein receptor proteins to cholesterol-rich lipid rafts in pancreatic alpha-cells: effects on glucagon stimulus-secretion coupling. Endocrinology 148:2157-2167.

Xia F, Xie L, Mihic A, Gao X, Chen Y, Gaisano HY, Tsushima RG (2008) Inhibition of cholesterol biosynthesis impairs insulin secretion and voltage-gated calcium channel function in pancreatic beta-cells. Endocrinology 149:5136-5145.

Xu J, Tse FW (1999) Brefeldin A increases the quantal size and alters the kinetics of catecholamine release from rat adrenal chromaffin cells. J Biol Chem 274:19095-19102.

Xu J, Xu Y, Ellis-Davies GC, Augustine GJ, Tse FW (2002) Differential regulation of exocytosis by $\alpha$ - and $\beta$-SNAPs. J Neurosci 22:53-61.

Xu J, Tang KS, Lu VB, Weerasinghe CP, Tse A, Tse FW (2005) Maintenance of quantal size and immediately releasable granules in rat chromaffin cells by glucocorticoid. Am J Physiol Cell Physiol 289:C1122-C1133.

Zamir O, Charlton MP (2006) Cholesterol and synaptic transmitter release at crayfish neuromuscular junctions. J Physiol 571:83-99.

Zhang J, Xue R, Ong WY, Chen P (2009) Roles of cholesterol in vesicle fusion and motion. Biophys J 97:1371-1380. 